\title{
The QCD pomeron in ultraperipheral heavy ion collisions: III. Photonuclear production of heavy quarks
}

\author{
V.P. Gonçalves ${ }^{1}$, M.V.T. Machado ${ }^{1,2}$ \\ 1 Instituto de Física e Matemática, Universidade Federal de Pelotas, Caixa Postal 354, CEP 96010-090, Pelotas, RS, Brazil \\ 2 High Energy Physics Phenomenology Group, GFPAE, IF-UFRGS, Caixa Postal 15051, CEP 91501-970, Porto Alegre, RS, \\ Brazil
}

Received: 11 August 2003 / Revised version: 3 September 2003 /

Published online: 10 October 2003 - (c) Springer-Verlag / Società Italiana di Fisica 2003

\begin{abstract}
We calculate the photonuclear production of heavy quarks in ultraperipheral heavy ion collisions. The integrated cross section and the rapidity distribution are computed employing sound high energy QCD formalisms like the collinear and semihard approaches as well as the saturation model. In particular, the color glass condensate (CGC) formalism is also considered using a simple phenomenological parameterization for the color field correlator in the medium, which allows us to obtain more reliable estimates for charm and bottom production at LHC energies.
\end{abstract}

\section{Introduction}

The recent results from RHIC suggest that relativistic heavy ion collisions at high energies probe QCD in the non-linear regime of high parton density [1]. In such a regime the growth of parton distributions should saturate, possibly forming a color glass condensate [2] (for a pedagogical presentation see $[3,4]$ ), which is characterized by a bulk momentum scale $Q_{\mathrm{s}}$. In particular, the RHIC data on the multiplicity distribution of the produced hadrons as a function of centrality, rapidity, and collision energy appears to be consistent with the CGC predictions. However, there are still a number of open questions, mainly associated to the fact that other models based on different assumptions reasonably describe the same set of experimental data $[5,6]$. For instance, the recent RHIC data indicate the lack of suppression in the high- $p_{\mathrm{T}}$ spectra of charged hadrons produced in $d+\mathrm{Au}$ collisions [7], in contrast to the initial expectation of the CGC formalism [1]. The main uncertainty present in those analyses is directly connected with the poor knowledge of the initial conditions of the heavy ion collisions. Theoretically, the early evolution of these nuclear collisions is governed by the dominant role of gluons [8], due to their large interaction probability and the large gluonic component in the initial nuclear wave functions. Consequently, a systematic measurement of the nuclear gluon distribution is of fundamental interest to understand the parton structure of nuclei, determine the initial conditions of the QGP and constrain the QCD dynamics at high energies.

In the last years, there has been a lot of interest in the description of electron-nucleus collisions at high energies, with particular emphasis on the behavior of the nu- clear structure functions [9] and their logarithmic slope [10] at small value of the Bjorken variable $x$, obtaining predictions which agree with the scarce experimental data. Moreover, the high energy heavy quark photoproduction on nuclei targets has been studied in detail in [11,12], considering the several scenarios for the QCD dynamics at high energies available. The results of those analyses show that future electron-nucleus colliders at HERA and RHIC $[13,14]$ probably could determine whether parton distributions saturate and constrain the behavior of the nuclear gluon distribution in the full kinematical range. However, until these colliders become reality we need to consider alternative searches in the current and/or scheduled accelerators which allow us to constrain the QCD dynamics. Here, we analyze the possibility of using ultraperipheral heavy ion collisions as a photonuclear collider and study the heavy quark production assuming distinct approaches for the QCD evolution.

The analysis of heavy quark production in ultraperipheral heavy ion collisions has been proposed many years ago [15], improved in [16-18] and recently revisited in [19-21]. Most of these approaches calculate the cross section assuming the validity of the collinear factorization, where the cross sections involving incoming hadrons are given, at all orders, by the convolution of intrinsically nonperturbative, but universal, quantities - the parton densities, with perturbatively calculable hard matrix elements, which are process dependent. In this approach all partons involved are assumed to be on mass shell, carrying only longitudinal momenta, and their transverse momenta are neglected in the QCD matrix elements. However, in the large energy (small- $x$ ) limit, the effects of the finite transverse momenta of the incoming partons become important, 
and the factorization must be generalized, implying that the cross sections are now $k_{\perp}$-factorized into an off-shell partonic cross section and a $k_{\perp}$-unintegrated parton density function $\mathcal{F}\left(x, k_{\perp}\right)$, characterizing the $k_{\perp}$-factorization approach [22-24]. The function $\mathcal{F}$ is obtained as a solution of the evolution equations associated to the dynamics that governs the QCD at high energies. Here, we estimate, for the first time, the total cross section and the rapidity dependence of the nuclear photoproduction of heavy quarks in ultraperipheral heavy ion collisions, considering the $k_{\perp}$-factorization approach and distinct nuclear unintegrated gluon distributions. Moreover, we extend the previous study for heavy quark production in the color glass condensate formalism considering a realistic photon flux and a phenomenological dipole cross section which is energy dependent, allowing one to obtain reliable estimates for the rapidity distribution within this formalism. For comparison, we also present the predictions for the cross section from the collinear factorization approach.

This paper is organized as follows. In the next section we present a brief review of ultraperipheral heavy ion collisions and write down the main formulas describing the photonuclear process in these collisions. In Sect. 3 we discuss some sound models for the heavy quark photoproduction in the collinear and $k_{\perp}$-factorization approaches. Moreover, we discuss our main assumptions in order to extend the previous results on the color glass condensate formalism. Finally, in Sect. 4 we present our results for the total cross section and rapidity distribution of charm and bottom production for the LHC energies.

\section{Ultraperipheral relativistic heavy ion collisions}

In heavy ion collisions the large number of photons coming from one of the colliding nuclei will allow one to study photoproduction, with energies $W_{\gamma N}$ reaching $950 \mathrm{GeV}$ for the LHC. The photonuclear cross sections are given by the convolution between the photon flux from one of the nuclei and the cross section for photon-nuclei scattering. The photon flux is given by the Weizsäcker-Williams method [25]. The flux from a charge $Z$ nucleus a distance $b$ away is

$$
\frac{\mathrm{d}^{3} N\left(\omega, b^{2}\right)}{\mathrm{d} \omega \mathrm{d}^{2} b}=\frac{Z^{2} \alpha_{\mathrm{em}} \eta^{2}}{\pi^{2} \omega b^{2}}\left[K_{1}^{2}(\eta)+\frac{1}{\gamma_{\mathrm{L}}^{2}} K_{0}^{2}(\eta)\right]
$$

where $\gamma_{\mathrm{L}}$ is the Lorentz boost of a single beam and $\eta=$ $\omega b / \gamma_{\mathrm{L}} ; K_{0}(\eta)$ and $K_{1}(\eta)$ are the modified Bessel functions. The requirement that photoproduction is not accompanied by a hadronic interaction (ultraperipheral collision) can be done by restricting the impact parameter $b$ to have values larger than twice the nuclear radius, $R_{A}=1.2 A^{1 / 3} \mathrm{fm}$. Therefore, the total photon flux interacting with the target nucleus is given by (1) integrated over the transverse area of the target for all impact parameters subject to the constraint that the two nuclei do not interact hadronically. An analytic approximation for $A A$ collisions can be obtained using as integration limit $b>2 R_{A}$, producing

$$
\begin{aligned}
& \frac{\mathrm{d} N(\omega)}{\mathrm{d} \omega}=\frac{2 Z^{2} \alpha_{\mathrm{em}}}{\pi \omega} \\
& \times\left[\bar{\eta} K_{0}(\bar{\eta}) K_{1}(\bar{\eta})+\frac{\bar{\eta}^{2}}{2}\left(K_{1}^{2}(\bar{\eta})-K_{0}^{2}(\bar{\eta})\right)\right],
\end{aligned}
$$

where $\bar{\eta}=2 \omega R_{A} / \gamma_{\mathrm{L}}$. The final expression for the production of heavy quarks in ultraperipheral heavy ion collisions is then given by

$$
\begin{aligned}
& \sigma_{A A \rightarrow Q \bar{Q} X}\left(\sqrt{S_{N N}}\right) \\
& =\int_{\omega_{\min }}^{\infty} \mathrm{d} \omega \frac{\mathrm{d} N(\omega)}{\mathrm{d} \omega} \sigma_{\gamma A \rightarrow Q \bar{Q} X}\left(W_{\gamma A}^{2}=2 \omega \sqrt{S_{N N}}\right),
\end{aligned}
$$

where $\omega_{\min }=M_{Q \bar{Q}}^{2} / 4 \gamma_{\mathrm{L}} m_{p}$ and $\sqrt{S_{N N}}$ is the CMS energy of the nucleus-nucleus system. The Lorentz factor for LHC is $\gamma_{\mathrm{L}}=2930$, giving the maximum CMS $\gamma N$ energy $W_{\gamma A} \lesssim 950 \mathrm{GeV}$. It is worth mentioning that the difference between the complete numeric and the analytical calculation presented above for the photon flux is less than $15 \%$ for most purposes [25].

Before considering the distinct models for the photonnucleus cross section, it is interesting to determine the values of $x$ which will be probed in ultraperipheral heavy ion collisions. The Bjorken $x$ variable is given by $x=$ $\left(M_{Q \bar{Q}} / 2 p\right) \mathrm{e}^{-y}$, where $M_{Q \bar{Q}}$ is the invariant mass of the photon-gluon system and $y$ the center of momentum rapidity. For $\mathrm{Pb}+\mathrm{Pb}$ collisions at LHC energies the nucleon momentum is equal to $p=2750 \mathrm{GeV}$; hence $x=$ $\left(M_{Q \bar{Q}} / 5500 \mathrm{GeV}\right) \mathrm{e}^{-y}$. Therefore, the region of small mass and large rapidities probes directly the high energy (small $x$ ) behavior of the QCD dynamics present in the $\gamma A$ cross section. This demonstrates that ultraperipheral heavy ion collisions at LHC represent a very good tool to constrain the high energy regime of the QCD dynamics, as already verified for two-photon processes [26-28].

\section{Models for nuclear heavy quark production}

For our further analysis of photonuclear production of heavy quarks we will consider distinct available high energy approaches. First, we take into account the usual collinear approach, where the production cross section is driven by the collinear gluon distribution on the nuclei. This contains a lot of information about nuclear shadowing, EMC, and anti-shadowing effects. Second, the $\boldsymbol{k}_{\perp}$-factorization formalism is introduced, where the relevant quantity is now the nuclear unintegrated gluon distribution. For this purpose, we analyze two simple parameterizations for it which are consistent with the data description on inclusive and diffractive DIS. Finally, we take into account the color glass condensate formalism, where the scattering process is viewed as the interaction of the probe particles with the strong nuclear color field treated in a classical approximation. The main quantity is the correlator of two Wilson 
lines, which is related to the dipole cross section and at lowest order has no energy dependence. We present a simple phenomenological parameterization which introduces higher orders corrections to the classical approximation and allows us to produce more realistic estimates for the cross section.

\subsection{The collinear approach}

In hard photon-hadron interactions the photon can behave as a point-like particle in the so-called direct photon processes or it can act as a source of partons, which then scatter against partons in the hadron, in the resolved photon processes (for a recent review see [29]). Resolved interactions stem from the photon fluctuation into a quarkantiquark state or a more complex partonic state, which both are embedded in the definition of the photon structure functions. Recently, the contribution of resolved photon processes in ultraperipheral heavy ion collisions was discussed in [20] and studied in detail in [21]. One of the main results is that at LHC, these contributions are $\approx 15$ and $20 \%$ of the total charm and bottom photoproduction cross sections, respectively; comparable to the shadowing effect. Here, we will consider only the direct photon contribution.

At high energies the main subprocess occurring when the photon probes the structure of the nucleus is the photon-gluon fusion producing the heavy quark pair. It can be described through perturbative QCD, with the cross section given in terms of the convolution between the elementary cross section for the subprocess $\gamma g \rightarrow Q \bar{Q}$ and the probability of finding a gluon inside the nucleus, namely the nuclear gluon distribution. In this collinear approach the heavy quark photoproduction cross section is given by

$$
\sigma_{\gamma A \rightarrow Q \bar{Q}}\left(W_{\gamma A}\right)=\int_{4 m_{Q}^{2}}^{W_{\gamma A}^{2}} \mathrm{~d} M_{Q \bar{Q}}^{2} \frac{\mathrm{d} \sigma_{\gamma g \rightarrow Q \bar{Q}}}{\mathrm{~d} M_{Q \bar{Q}}^{2}} g_{A}\left(x, \mu^{2}\right),
$$

where the quantity $\mathrm{d} \sigma_{Q \bar{Q}} / \mathrm{d} M_{Q \bar{Q}}^{2}$ is calculable perturbatively, and $M_{Q \bar{Q}}$ is the invariant mass of the heavy quark pair with $x=M_{Q \bar{Q}}^{2} / W_{\gamma A}^{2}$. The CMS energy of the $\gamma A$ system is labelled $W_{\gamma A}$, and $g_{A}\left(x, \mu^{2}\right)$ is the gluon density inside the nuclear medium, with $\mu$ being the factorization scale and $m_{Q}$ the heavy quark mass. For our purpose here we will use $\mu^{2}=4 m_{Q}^{2}$, with $m_{c}=1.5 \mathrm{GeV}$ and $m_{b}=4.5 \mathrm{GeV}$. The differential cross section in leading order is given by [30]

$$
\begin{aligned}
& \frac{\mathrm{d} \sigma_{\gamma g \rightarrow Q \bar{Q}}}{\mathrm{~d} M_{Q \bar{Q}}}=\frac{4 \pi \alpha_{\mathrm{em}} \alpha_{s}\left(\mu^{2}\right) e_{Q}^{2}}{M_{Q \bar{Q}}^{2}} \\
& \times\left[\left(1+\beta+\frac{1}{2} \beta^{2}\right) \ln \left(\frac{1+\sqrt{1-\beta}}{1-\sqrt{1-\beta}}\right)-(1+\beta) \sqrt{1-\beta}\right]
\end{aligned}
$$

where $e_{Q}$ is the heavy quark charge and $\beta=4 m_{Q}^{2} / M_{Q \bar{Q}}^{2}$. In our further calculation in the collinear approach one considers that $x g_{A}\left(x, Q^{2}\right)=R_{g}\left(x, Q^{2}\right) \times x g_{N}\left(x, Q^{2}\right)$, where the $R_{g}$ parameterize the medium effects as proposed in [31] and $x g_{N}$ is the nucleon gluon distribution given by the GRV98(LO) parameterization [32]. It is worth mentioning that different choices for the factorization scale and quark mass produce a distinct overall normalization to the total cross section at photon-nucleus and ultraperipheral nucleus-nucleus interactions. For details see [12], where the heavy quark photoproduction at eRHIC and THERA energies has been discussed. In Sect. 4 we discuss the dependence of our results on the choice of the quark mass and parton distribution parameterization.

\subsection{The $k_{\perp}$-factorization formalism}

In the $k_{\perp}$-factorization (or semihard) approach, the relevant QCD diagrams are considered with the virtualities and polarizations of the initial partons, carrying information on their transverse momenta. The scattering processes are described through the convolution of off-shell matrix elements with the unintegrated parton distribution, $\mathcal{F}\left(x, \boldsymbol{k}_{\perp}\right)$ (see [33] for a review). The latter can recover the usual parton distributions in the double logarithmic limit by its integration over the transverse momentum of the $\boldsymbol{k}_{\perp}$ exchanged gluon. The gluon longitudinal momentum fraction is related to the CMS energy, $W_{\gamma}$, in the heavy quark photoproduction case by $x=4 m_{Q}^{2} / W_{\gamma A}^{2}$, as in the collinear case. The cross section for the heavy quark photoproduction process is given by the convolution of the unintegrated gluon function with the off-shell matrix elements [33-36]. Considering only the direct component of the photon we see that $\sigma_{\text {tot }}^{\text {phot }}$ reads [35]

$$
\begin{aligned}
& \sigma_{\text {tot }}^{\text {phot }}\left(W_{\gamma A}\right) \\
& =\frac{\alpha_{\mathrm{em}} e_{Q}^{2}}{\pi} \int \mathrm{d} z \mathrm{~d}^{2} \boldsymbol{p}_{1 \perp} \mathrm{d}^{2} \boldsymbol{k}_{\perp} \frac{\alpha_{s}\left(\mu^{2}\right) \mathcal{F}\left(x, \boldsymbol{k}_{\perp}^{2} ; \mu^{2}\right)}{\boldsymbol{k}_{\perp}^{2}} \\
& \times\left\{\left[z^{2}+(1-z)^{2}\right]\left(\frac{\boldsymbol{p}_{1 \perp}}{D_{1}}+\frac{\left(\boldsymbol{k}_{\perp}-\boldsymbol{p}_{1 \perp}\right)}{D_{2}}\right)^{2}\right. \\
& \left.\quad+m_{Q}^{2}\left(\frac{1}{D_{1}}+\frac{1}{D_{2}}\right)^{2}\right\}
\end{aligned}
$$

where $D_{1} \equiv \boldsymbol{p}_{1 \perp}^{2}+m_{Q}^{2}$ and $D_{2} \equiv\left(\boldsymbol{k}_{\perp}-\boldsymbol{p}_{1 \perp}\right)^{2}+m_{Q}^{2}$. The transverse momenta of the heavy quark (antiquark) are denoted by $\boldsymbol{p}_{1 \perp}$ and $\boldsymbol{p}_{2 \perp}=\left(\boldsymbol{k}_{\perp}-\boldsymbol{p}_{1 \perp}\right)$, respectively. The heavy quark longitudinal momentum fraction is labeled by $z$. The scale $\mu$ in the strong coupling constant in general is taken to be equal to the gluon virtuality, in close connection with the BLM scheme [37]. Here, we will use the prescription $\mu^{2}=\boldsymbol{k}_{\perp}^{2}+m_{Q}^{2}$.

In order to perform a phenomenological analysis within the $k_{\perp}$-factorization approach, in the following we use two distinct parameterizations for the unintegrated gluon distribution (for details see [12]). First, one considers the derivative of the collinear nuclear gluon parton distribution function; this is quite successful in the proton case 
and tested in the nuclear case in [12]. It simply reads

$$
\mathcal{F}_{\text {nuc }}\left(x, \boldsymbol{k}_{\perp}^{2} ; A\right)=\frac{\partial x G_{A}\left(x, \boldsymbol{k}_{\perp}^{2}\right)}{\partial \ln \boldsymbol{k}_{\perp}^{2}},
$$

where $x G_{A}\left(x, Q^{2}\right)$ is the nuclear gluon distribution, which was taken from the EKS parameterization [31] for the medium effects and the GRV94(LO) for the nucleon parton distribution [38]. The latter choice is supported by the good description of heavy quark photoproduction in the full kinematical region [12]. As a consequence, with this procedure we include in our calculations the medium effects (shadowing, anti-shadowing, EMC and Fermi motion effects) estimated by that parameterization. Moreover, we emphasize that this nuclear gluon distribution is a solution of the DGLAP evolution equations, which is associated to a linear dynamics that does not consider dynamical saturation effects.

The second parameterization is given by the model introduced in [39], which provides an extension of the $e p$ saturation model through the Glauber-Gribov formalism. In this model the cross section for the heavy quark photoproduction on nuclei targets is given by $[11,39]$

$$
\begin{aligned}
& \sigma_{\text {tot }}^{\gamma A}(W, A) \\
& =\int_{0}^{1} \mathrm{~d} z \int \mathrm{d}^{2} \boldsymbol{r}\left|\Psi_{\mathrm{T}}\left(z, \boldsymbol{r}, Q^{2}=0\right)\right|^{2} \sigma_{\text {dip }}^{A}\left(\tilde{x}, \boldsymbol{r}^{2}, A\right),
\end{aligned}
$$

where the transverse wave function is known (see e.g. [40]). As $\left|\Psi_{\mathrm{L}}\right|^{2} \propto Q^{2}$, the longitudinal piece does not contribute for $Q^{2}=0$. The nuclear dipole cross section is given by

$$
\begin{aligned}
& \sigma_{\text {dip }}^{A}\left(\tilde{x}, \boldsymbol{r}^{2}, A\right) \\
& =\int \mathrm{d}^{2} b 2\left\{1-\exp \left[-\frac{1}{2} A T_{A}(b) \sigma_{\text {dip }}^{\mathrm{p}}\left(\tilde{x}, \boldsymbol{r}^{2}\right)\right]\right\},
\end{aligned}
$$

where $b$ is the impact parameter of the center of the dipole relative to the center of the nucleus, and the integrand gives the total dipole-nucleus cross section for fixed impact parameter. The nuclear profile function is labelled by $T_{A}(b)$, which will be obtained from the three-parameter Fermi distribution for the nuclear density [41]. The parameterization for the dipole cross section takes the eikonal-like form, $\sigma_{\text {dip }}^{\mathrm{p}}\left(\tilde{x}, \boldsymbol{r}^{2}\right)=\sigma_{0}\left[1-\exp \left(-Q_{\mathrm{s}}^{2}(\tilde{x}) \boldsymbol{r}^{2} / 4\right)\right]$, where one has used the parameters from [42], which include the charm quark with mass $m_{c}=1.5 \mathrm{GeV}$ and the definition $\tilde{x}=\left(Q^{2}+4 m_{Q}^{2}\right) / W_{\gamma A}^{2}$. The saturation scale $Q_{\mathrm{s}}^{2}(x)=$ $\left(x_{0} / x\right)^{\lambda} \mathrm{GeV}^{2}$ gives the onset of the saturation phenomenon for the process.

The equation above sums up all the multiple elastic rescattering diagrams of the $q \bar{q}$ pair and is justified for a large coherence length, where the transverse separation $r$ of partons in the multiparton Fock state of the photon becomes as good a conserved quantity as the angular momentum, namely the size of the pair $r$ becomes an eigenvalue of the scattering matrix. The corresponding unintegrated gluon distribution can be recovered from a Bessel-Fourier transform to the momentum representation [39],

$$
\begin{aligned}
& \mathcal{F}_{\text {nuc }}\left(x, \boldsymbol{k}_{\perp}^{2}, b\right)=\frac{N_{c}}{\pi^{2} \alpha_{\mathrm{s}}}\left(\frac{\boldsymbol{k}_{\perp}^{2}}{Q_{\mathrm{s}}^{2}}\right) \\
& \times \sum_{m=1}^{\infty} \sum_{n=0}^{m} \frac{\left(-\frac{1}{2} A T_{A}(b) \sigma_{0}\right)^{m}}{m !} C_{m}^{n} \frac{(-1)^{n}}{n} \exp \left(-\frac{\boldsymbol{k}_{\perp}^{2}}{n Q_{\mathrm{s}}^{2}}\right)
\end{aligned}
$$

which depends on the transverse momentum $\boldsymbol{k}_{\perp}$ through the scaling variable $\tau \equiv \boldsymbol{k}_{\perp}^{2} / Q_{\mathrm{s}}^{2}$. The unintegrated gluon vanishes asymptotically at $\boldsymbol{k}_{\perp}^{2} \rightarrow 0, \infty$ and its maximum can be identified with the saturation scale $Q_{\mathrm{s} A}(x)[39,43]$. The model recovers the original one for the proton case, taking $A=1$ and the normalization condition $\int \mathrm{d}^{2} b T_{A}(b)=1$.

In [11], it was verified that the resummation at the proton level is less sizeable in the final results at nuclear level. Therefore, this fact allows us to take just the color transparency behavior on the dipole-nucleon cross section. Hence, in such a particular case one can compute analytically the unintegrated gluon distribution, which is expressed as

$$
\mathcal{F}_{\text {nuc }}\left(x, \boldsymbol{k}_{\perp}^{2}, b\right)=\frac{N_{c}}{2 \alpha_{\mathrm{s}} \pi^{2}}\left(\frac{\boldsymbol{k}_{\perp}^{2}}{Q_{\mathrm{s} A}^{2}(x)}\right) \exp \left(-\frac{\boldsymbol{k}_{\perp}^{2}}{Q_{\mathrm{s} A}^{2}(x)}\right),
$$

where $Q_{\mathrm{s} A}^{2}(x)=\frac{1}{2} A T_{A}(b) \sigma_{0} Q_{\mathrm{s}}^{2}(x)$ define the nuclear saturation scale. Such an approximation is justified in the heavy quark case, which is dominated by small dipole configurations (a large transverse momentum $\boldsymbol{k}_{\perp}^{2} \simeq m_{Q}^{2}$ ). It is clear that (11) presents a scaling pattern on the variable $\tau=\boldsymbol{k}_{\perp}^{2} / Q_{\mathrm{s} A}^{2}$, which implies scaling on $\tau$ in the nuclear heavy quark production. Recently, this feature has been shown also in the nucleon case [44].

Some comments are in order here. In our analysis we have disregarded higher-order Fock states in the photon wave function and considered only the evolution in the dipole cross section. In principle, at leading order and for inclusive processes this is a reasonable approximation. However, for diffractive processes, for example, the $q \bar{q} g$ component cannot be disregarded. The recent results for the NLO corrections for the impact factor [45] will allow one to verify the validity of the color dipole approach at higher orders. Another point which deserves discussion is that we have assumed the validity of the $k_{\perp}$-factorization in photon-nucleus interactions. At high energies and intermediate densities, this is a reasonable assumption, since the derivation of the $k_{\perp}$-factorization presented in [46] can be directly extended for the nuclear heavy quark photoproduction. However, for very large parton densities a breakdown of the $k_{\perp^{-}}$(and collinear) factorization is expected, mainly associated to the effects of the non-linearity of the non-Abelian gluon field $[2,47,48]$. Finally, we also have disregarded the resolved photon contribution in the semihard approach. For completeness, let us quote the estimates for the resolved component on the nucleon level. In [36] it gives a contribution of order $20-30 \%$, rising faster with energy (hence important mostly at large rapidities) than the direct photon component and being stable under different choices for the unintegrated gluon distributions and 
quark mass. Moreover, the resolved contribution is somewhat higher than in the collinear approach due to the non-zero transverse momentum transfer effect. We expect a similar trend in photon-nucleus interactions.

\subsection{The color glass condensate formalism}

At small $x$ and/or large $A$ one expects the transition of the regime described by the linear dynamics (DGLAP, BFKL) (for a review, see e.g. [49]), where only the parton emissions are considered, to a new regime where the physical process of recombination of partons becomes important in the parton cascade and the evolution is given by a non-linear evolution equation. In this regime a color glass condensate (CGC) is expected to be formed [2], being characterized by the limitation on the maximum phase-space parton density that can be reached in the hadron/nuclear wave function (parton saturation) and very high values of the QCD field strength $F_{\mu \nu} \approx 1 / \sqrt{\alpha_{\mathrm{s}}}$ [50]. The large values of the gluon distribution at saturation (large occupation number of the soft gluon modes) suggests the use of semi-classical methods, which allow one to describe the small- $x$ gluons inside a fast moving nucleus by a classical color field. This color field is driven by a classical Yang-Mills equation whose source term is provided by faster partons. When the energy further increases, the structure of the classical field equations does not change, but only the correlations of the color source. This change can be computed in perturbation theory and expressed as a functional renormalization group equation for the weight function, in which the "fast" partons are integrated out in steps of rapidity and in the background of the classical field generated at the previous step. This approach enables one to calculate cross sections in a high gluon density environment. Recently, this approach has been applied for the $e A[47,51], p A[47,52,53]$ and $A A$ processes [48].

In [19] the heavy quark production in ultraperipheral heavy ion collisions has been analyzed in the color glass condensate formalism. In particular, those authors have considered the photon-nuclei interaction, taking into account the electromagnetic interaction to lowest order in the coupling and the interactions with the strong color background field to all orders. The quantum evolution is not included in the calculations. Their prediction for the rapidity ( $y$ ) distribution of the heavy quark (or antiquark) is given by

$$
\begin{gathered}
\frac{\mathrm{d} \sigma_{A A \rightarrow Q \bar{Q} X}}{\mathrm{~d} y}=\pi R_{A}^{2} \frac{N_{c}\left(Z \alpha_{\mathrm{em}}\right)^{2} e_{q}^{2}}{6 \pi^{4}} \int_{2 R_{A}}^{\frac{\gamma_{\mathrm{L}}}{m_{Q}}} \frac{\mathrm{d}^{2} \boldsymbol{b}}{\boldsymbol{b}^{2}} \int_{0}^{+\infty} \mathrm{d} \boldsymbol{k}_{\perp}^{2} C\left(\boldsymbol{k}_{\perp}\right) \\
\quad \times\left\{1+\frac{4\left(\boldsymbol{k}_{\perp}^{2}-m_{Q}^{2}\right)}{k_{\perp} \sqrt{\boldsymbol{k}_{\perp}^{2}+4 m_{Q}^{2}}} \operatorname{arcth} \frac{k_{\perp}}{\sqrt{\boldsymbol{k}_{\perp}^{2}+4 m_{Q}^{2}}}\right\}, \quad(12)
\end{gathered}
$$

with $N_{c}$ being the color number and $e_{q}$ the quark charge. The color field correlator $C\left(\boldsymbol{k}_{\perp}\right)$ in the medium is given by

$$
C\left(\boldsymbol{k}_{\perp}\right) \equiv \int \mathrm{d}^{2} \boldsymbol{r} \mathrm{e}^{\mathrm{i} \boldsymbol{k}_{\perp} \cdot \boldsymbol{r}} \mathrm{e}^{-B_{2}(\boldsymbol{r})}
$$

$$
=\int \mathrm{d}^{2} \boldsymbol{r} \mathrm{e}^{\mathrm{i} \boldsymbol{k}_{\perp} \cdot \boldsymbol{r}}\left\langle U(0) U^{\dagger}(\boldsymbol{r})\right\rangle_{\rho}
$$

with $\langle\ldots\rangle_{\rho}$ representing the average over all configurations of the color fields in the nucleus. The unitarity matrix $U(\boldsymbol{r})$ contains the information related to the interactions between the quark and the colored glass condensate (classical color field of the nucleus) and is expressed in terms of the color sources in the nucleus. Therefore, $C\left(\boldsymbol{k}_{\perp}\right)$ depends on the structure of the color sources describing the target nucleus, and it describes the interactions of a high energy probe with the target.

In [19] the McLerran-Venugopalan model for the correlator $C\left(\boldsymbol{k}_{\perp}\right)$ was considered. In this model the function $B_{2}(\boldsymbol{r})$ in (13) is approximated as follows:

$$
B_{2}(\boldsymbol{r}) \approx \frac{Q_{\mathrm{s} A}^{2} \boldsymbol{r}^{2}}{4 \pi} \ln \left(\frac{1}{\boldsymbol{r} \Lambda}\right),
$$

where the saturation scale $Q_{\mathrm{s} A}$ at this classical level does not depend on the rapidity (energy) and $\Lambda$ is an infrared cutoff related to the scale at which color neutrality occurs. In principle, $\Lambda$ is at least as large as $\Lambda_{\mathrm{QCD}}$. For a saturated target, it can be probed that color neutrality occurs over transverse spatial scales as small as $1 / Q_{\mathrm{s}}$ [54]. Let us summarize the results coming from applying the approximation above and $\Lambda=\Lambda_{\mathrm{QCD}}$ in (12). Supposing $Q_{\mathrm{s} A} / \Lambda_{\mathrm{QCD}}=10(y=2.3)$ one obtains $\mathrm{d} \sigma / \mathrm{d} y=355 \mathrm{mb}$ for charm and $\mathrm{d} \sigma / \mathrm{d} y=11 \mathrm{mb}$ for bottom with the rapidity distribution being flat on $y$.

In principle, extending the previous calculation to include the quantum evolution is just a matter of changing the $C\left(\boldsymbol{k}_{\perp}\right)$ present in the previous calculations by one that has been calculated considering the evolution of the sources. However, the general solution of the functional renormalization group equation for the weight function is not known, but only approximate solutions in some limiting kinematical regimes have been obtained [55]. In general, the quantum corrections lead to a modification of the distribution function of the hard sources when the energy increases, which implies a rapidity dependence for the correlator $C\left(\boldsymbol{k}_{\perp}\right)$.

In order to go further and introduce an energy dependence in the calculations one makes use of the fact that the function $C\left(\boldsymbol{k}_{\perp}\right)$ is directly related to the Fourier transform of the dipole-nucleus total cross section, as follows:

$$
\begin{aligned}
& C\left(x, \boldsymbol{k}_{\perp}\right) \equiv \\
& \frac{1}{2 \pi R_{A}^{2}} \int \mathrm{d}^{2} \boldsymbol{r} \mathrm{e}^{\mathrm{i} \boldsymbol{k}_{\perp} \cdot \boldsymbol{r}}\left[\sigma_{\mathrm{dip}}(x, \boldsymbol{r} \rightarrow \infty)-\sigma_{\mathrm{dip}}(x, \boldsymbol{r})\right] .
\end{aligned}
$$

For this definition of the correlator, we have a direct relation between this quantity and the unintegrated gluon density, given by $\mathcal{F}\left(x, \boldsymbol{k}_{\perp}\right)=\left(3 R_{A}^{2} / 8 \pi^{2} \alpha_{\mathrm{s}}\right) \boldsymbol{k}_{\perp}^{2} C\left(x, \boldsymbol{k}_{\perp}\right)$. In our analysis we will employ an educated guess for the dipole-nucleus cross section:

$$
\sigma_{\mathrm{dip}}^{A}(x, \boldsymbol{r})=2 \pi R_{A}^{2}\left[1-\exp \left(-\frac{Q_{\mathrm{s} A}^{2}(x) \boldsymbol{r}^{2}}{4}\right)\right],
$$



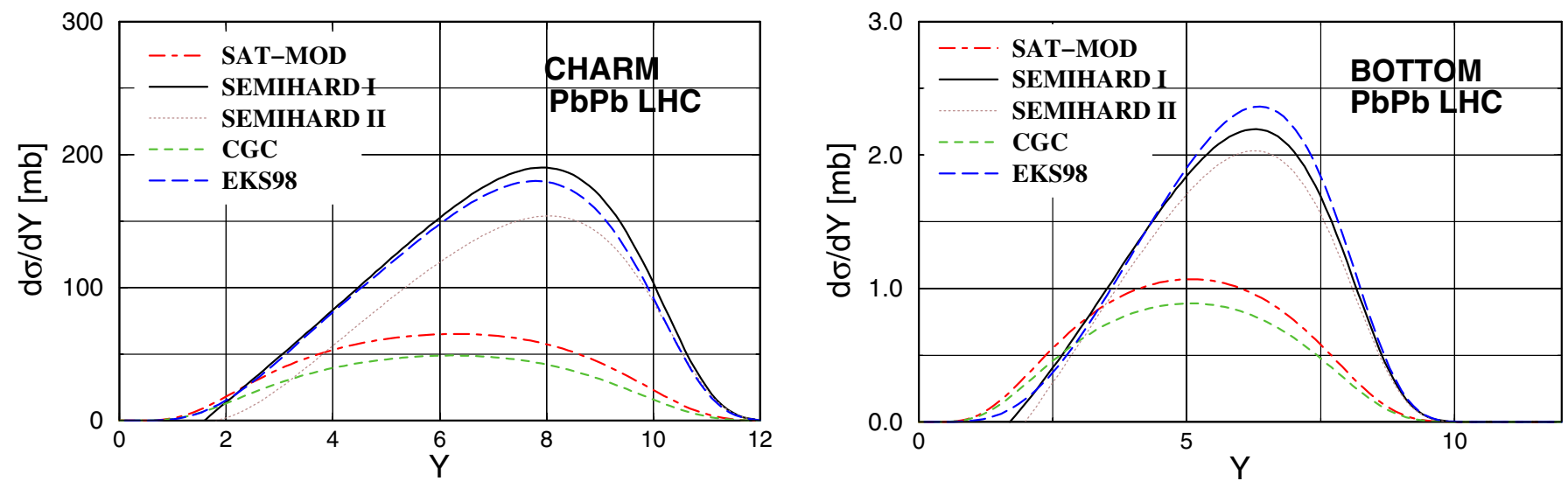

Fig. 1. The rapidity distribution for the distinct high energy approaches: collinear approach (long-dashed lines), semihard formalism (solid and dotted lines), saturation model (dot-dashed lines) and color glass condensate (dashed lines). The corresponding mass values are $m_{c}=1.5 \mathrm{GeV}$ and $m_{b}=4.5 \mathrm{GeV}$

where we assume that $Q_{\mathrm{s} A}^{2}(x)=A^{1 / 3} \times Q_{\mathrm{s}}^{2}(x)$. This model is inspired by the saturation model for the dipole-proton scattering proposed in [42], and encodes the main properties of the high density approaches, namely color transparency for small pair separations $(r \rightarrow 0)$ and saturation for large pair separations $(r \rightarrow \infty)$. Moreover, the nuclear $A$ dependence assumed for the saturation scale agrees with the recent analysis for the scattering of a small dipole in a nuclear target in the McLerran-Venugopalan model and fixed coupling BFKL dynamics [56]. However, the energy dependence of the saturation scale still is an open question. Recently, several groups have studied the solutions of the Balitski-Kovchegov equation $[57,58]$, with $x$ dependence for $Q_{\mathrm{s}}$ slightly different from the ansatz used here.

Embedding (16) in the definition of (15) we obtain the following analytical expression for the color field correlator:

$$
\widetilde{C}\left(x, \boldsymbol{k}_{\perp}\right)=\frac{4 \pi}{Q_{\mathrm{s} A}^{2}(x)} \exp \left(-\frac{\boldsymbol{k}_{\perp}^{2}}{Q_{\mathrm{s} A}^{2}(x)}\right),
$$

which also obeys the normalization condition

$$
\int \mathrm{d}^{2} \boldsymbol{k} \widetilde{C}\left(x, \boldsymbol{k}_{\perp}\right) /(2 \pi)^{2}=1 .
$$

In the next section, we compute the photonuclear cross section using the correlator above and in addition consider the complete photon flux instead of the approximation leading to (12). The final expression now reads

$$
\begin{aligned}
& \frac{\mathrm{d} \sigma_{A A \rightarrow Q \bar{Q} X}}{\mathrm{~d} Y}=\omega \frac{\mathrm{d} N(\omega)}{\mathrm{d} \omega} \frac{\alpha_{\mathrm{em}} e_{q}^{2}}{2 \pi^{2}} \int_{0}^{+\infty} \mathrm{d} \boldsymbol{k}_{\perp}^{2} \pi R_{A}^{2} \widetilde{C}\left(\boldsymbol{k}_{\perp}\right) \\
& \quad \times\left\{1+\frac{4\left(\boldsymbol{k}_{\perp}^{2}-m_{Q}^{2}\right)}{k_{\perp} \sqrt{\boldsymbol{k}_{\perp}^{2}+4 m_{Q}^{2}}} \operatorname{arcth} \frac{k_{\perp}}{\sqrt{\boldsymbol{k}_{\perp}^{2}+4 m_{Q}^{2}}}\right\},
\end{aligned}
$$

where we define the rapidity $Y \equiv \ln (1 / x)=\ln \left(2 \omega \gamma_{\mathrm{L}} / 4 m_{Q}^{2}\right)$ and the variable transformation between $\omega$ and $Y$ should be carried out. The equivalent photon flux is taken from (2).

\section{Results and discussions}

In this section we present the numerical calculation of the rapidity $Y$ distribution and total cross section for charm and bottom photonuclear production. In particular, we are focusing mostly on the LHC domain where small values of $x$ would be probed. At RHIC, $x=\left(M_{Q \bar{Q}} / 200 \mathrm{GeV}\right) \mathrm{e}^{-y}$, which implies $x>10^{-2}$ and, consequently, small deviations between the high energy QCD approaches. In the following, one considers the charm and bottom masses $m_{c}=1.5 \mathrm{GeV}$ and $m_{b}=4.5 \mathrm{GeV}$, respectively. Moreover, for $\mathrm{PbPb}(A=208)$ collisions at LHC, one has the CMS energy of the ion-ion system $\sqrt{S_{N N}}=5500 \mathrm{GeV}$ and the Lorentz factor $\gamma_{\mathrm{L}}=2930$.

In Fig. 1 are shown the rapidity distribution, for the distinct high energy approaches considered before. The collinear result is denoted by the long-dashed curves, where use has been made of (3) and (4) employing the EKS98 parameterization for the collinear nuclear gluon function. The solid and dotted lines label the semihard $\left(\boldsymbol{k}_{\perp}\right.$-factorization) results, where one has used (6) and the ansatz given by (7) for the unintegrated gluon function. Two possibilities for the nucleon gluon distribution are considered:

(I) GRV94(LO), the solid line, and

(II) GRV98(LO), the dotted line.

The saturation model results are denoted by the dotdashed lines, with the input given by (8). The color glass condensate prediction (dashed lines), is given by our phenomenological ansatz using expressions (17) and (18). The predictions for the collinear approach and the semihard formalism are similar for both charm and bottom production and give somewhat larger values than the saturation and CGC results. One possible interpretation for the similarity between the predictions of the semihard approach and the collinear one is that the expected enhancement in the $\boldsymbol{k}_{\perp}$-factorization formalism, associated to the resummation of the $\left(\alpha_{\mathrm{s}} \ln \frac{\sqrt{s}}{m_{Q}}\right)^{n}$ in the coefficient function [22], is not sizeable for inclusive quantities in the kinematic region of the future colliders. This feature entails the trend already verified at nucleon level as in photon-proton 
Table 1. The photonuclear heavy quark total cross sections for ultraperipheral heavy ion collisions at $\operatorname{LHC}\left(\sqrt{S_{N N}}=\right.$ $5500 \mathrm{GeV}$ ) for $\mathrm{PbPb}$

\begin{tabular}{lrrrr}
\hline $\bar{Q} \bar{Q}$ & Collinear & SAT-MOD & SEMIHARD I (II) & CGC \\
\hline$c \bar{c}$ & $2056 \mathrm{mb}$ & $862 \mathrm{mb}$ & $2079(1679.3) \mathrm{mb}$ & $633 \mathrm{mb}$ \\
$b \bar{b}$ & $20.1 \mathrm{mb}$ & $10.75 \mathrm{mb}$ & $18(15.5) \mathrm{mb}$ & $8.9 \mathrm{mb}$ \\
\hline
\end{tabular}

and photon-nuclei collisions [12]. Even inclusive cross sections in hadroproduction provide similar results between collinear and semihard (dipole) approaches [59]. Probably, a more promising quantity to clarify this issue would be the transverse momentum $\boldsymbol{p}_{\perp}$ distribution. In this case, the semihard approach seems to be in better agreement with experimental data in the $p p$ collisions than the collinear approach [34].

Our phenomenological ansatz within the CGC formalism gives similar results as the saturation model, but it should be noticed that the physical assumptions in those models are distinct. While (9) considers multiple scattering on single nucleons, our expression for the dipole-nucleus cross section [see (16)] assumes scattering on a black area filled by partons coming from many nucleons. It is important to emphasize that the current experimental data for the nuclear structure function can only be described if the first choice is implemented [39]. However, the correct expression for $\sigma_{\text {dip }}^{A}$ in the kinematical range of the future colliders is still an open question.

Let us now compute the integrated cross section considering the distinct models. The results are presented in Table 1 for charm and bottom pair production. The collinear approach gives a larger rate, followed by the semihard approach, a clear trend from the distribution on rapidity. The saturation model and CGC formalisms give similar results, including a closer ratio for charm to bottom production. Concerning the CGC approach, our phenomenological educated guess for the color field correlator seems to produce quite reliable estimates.

Let us estimate the uncertainties present in our predictions using the collinear approach. We find that for bottom production our prediction decreases by $\approx 20 \%$ if we assume $m_{b}=4.75 \mathrm{GeV}$ and by $\approx 10 \%$ if we assume that the factorization scale is $\mu^{2}=m_{b}^{2}$. Moreover, if the MRST gluon density [60] is used instead of the GRV98 parameterization, our predictions decrease by $\approx 10 \%$. For charm production, the differences are larger due to the small values of $x$ probed in that process. Our results for the total charm production cross section in the collinear approach are similar to those computed in [21]. However, they differ largely for bottom production, even using the same set of scales and parton distributions. We believe that our results are reliable, since our prediction for the photon-nucleon interaction is consistent with the HERA data [12], as well as with the simple expectation $\sigma_{\gamma A \rightarrow b \bar{b} X} \approx A \times \sigma_{\gamma p \rightarrow b \bar{b} X}$.

Regarding the semihard approach we have checked the uncertainties coming from the quark mass and different choices for the gluon parameterization as input for the unintegrated function. In comparison with the default value $m_{c}=1.5 \mathrm{GeV}$ for charm, we have an enhancement of $\approx 35 \%$ using $m_{c}=1.2 \mathrm{GeV}$, whereas the result decreases by the same amount for $m_{c}=1.8 \mathrm{GeV}$ (similar results hold for bottom). Considering the default value for the quark mass, the uncertainty when using the GRV98 parameterization is of order $\approx 20 \%$. In Table 1 we provide the cross section using

(I) the GRV94 and

(II) the GRV98 parameterization. There is an additional uncertainty coming from the energy scale $\mu^{2}$ entering on the strong coupling constant. Here we have used the optimal choice, giving correct results at the nucleon level and allowing a simple translation to occur to the dipole (position space) representation, since the energy scale does not depend on the quark transverse momentum.

In order to check if the differences between the saturation approach and CGC come from the integration weights in (6) and (18) or from a different mass number dependence for the saturation scales, we have used our ansatz (16) in the calculation of the photonuclear cross section. We have found that for lead the CGC ansatz gives a result $\approx 40 \%$ lower than the saturation model, whereas for calcium the results are almost identical. Therefore, as the deviation between saturation model and CGC comes mostly from the different definition for the $A$ dependence of $Q_{\mathrm{s}} A(x)$, an experimental analysis of this process for different nuclei can be useful.

In conclusion, the photonuclear production of heavy quarks allow us to constrain already in the current nuclear accelerators the QCD dynamics since the main features from photon-nuclei collisions hold in the coherent ultraperipheral reactions. As a summary, we have computed the photonuclear production of heavy quarks in ultraperipheral heavy ion collisions. One obtains the integrated cross section and the rapidity distribution through well established QCD approaches, namely the collinear and semihard factorization formalisms as well as the saturation model. For the first time, quantitative predictions for the latter two approaches are presented, whereas previous collinear calculations are consistently corroborated. Moreover, the color glass condensate formalism has been considered using a simple educated guess for the color field correlator in the medium, which allowed us to reach reliable estimates at LHC energies.

Acknowledgements. M.V.T.M. thanks the support of the High Energy Physics Phenomenology Group at the Institute of Physics, GFPAE IF-UFRGS, Porto Alegre. This work was partially financed by the Brazilian funding agencies $\mathrm{CNPq}$ and FAPERGS.

\section{References}

1. D. Kharzeev, M. Nardi, Phys. Lett. B 507, 121 (2001); D. Kharzeev, E. Levin, Phys. Lett. B 523, 79 (2001); D. Kharzeev, E. Levin, L. McLerran, Phys. Lett. B 561, 93 (2003) 
2. E. Iancu, A. Leonidov, L. McLerran, Nucl. Phys. A 692 , 583 (2001); E. Ferreiro, E. Iancu, A. Leonidov, L. McLerran, Nucl. Phys. A 703, 489 (2002)

3. E. Iancu, A. Leonidov, L. McLerran, hep-ph/0202270

4. E. Iancu, R. Venugopalan, hep-ph/0303204

5. K.J. Eskola, Nucl. Phys. A 698, 78 (2002)

6. N. Armesto, C. Pajares, Int. J. Mod. Phys. A 15, 2019 (2000)

7. PHOBOS Collaboration, B. Back et al., Phys. Rev. Lett. 91, 072302 (2003); PHENIX Collaboration, S. Adler et al., Phys. Rev. Lett. 91, 072303 (2003); STAR Collaboration, J. Adams et al., Phys. Rev. Lett. 91, 072304 (2003); BRAHMS Collaboration, I. Arsen et al., Phys. Rev. Lett. 91, 072305 (2003)

8. K. Geiger. Phys. Rep. 258, 237 (1995); X.-N Wang. Phys. Rep. 280, 287 (1997)

9. A.L. Ayala, M.B. Gay Ducati, E.M. Levin, Nucl. Phys. B 493, 305 (1997); Z. Huang, H.J. Lu, I. Sarcevic, Nucl. Phys. A 637, 79 (1998); M.B. Gay Ducati, V.P. Gonçalves, Phys. Rev. C 60, 058201 (1999); N. Armesto et al., Eur. Phys. J. C 29, 531 (2003); H. Kowalski, D. Teaney, hep$\mathrm{ph} / 0304189$

10. M.B. Gay Ducati, V.P. Gonçalves, Phys. Lett. B 466, 375 (1999); V.P. Gonçalves, Phys. Lett. B 495, 303 (2000)

11. V.P. Gonçalves, M.V. Machado, Eur. Phys. J. C 30, 387 (2003)

12. V.P. Gonçalves, M.V. Machado, hep-ph/0307129

13. M. Arneodo et al., in Proceedings of the workshop on Future Physics at HERA, edited by G. Ingelman, A. De Roeck, R. Klanner, vol. 2, pp. 887-926 (1996)

14. R. Venugopalan, AIP Conf. Proc. 588, 121 (2001), hep$\mathrm{ph} / 0102087$

15. Ch. Hofmann, G. Soff, A. Schafer, W. Greiner, Phys. Lett. B 262, 210 (1991)

16. N. Baron, G. Baur, Phys. Rev. C 48, 1999 (1993)

17. M. Greiner, M. Vidovic, Ch. Hofman, A. Schafer, G. Soff, Phys. Rev. C 51, 911 (1995)

18. F. Krauss, M. Greiner, G. Soff, Prog. Part. Nucl. Phys. 39, 503 (1997)

19. F. Gelis, A. Peshier, Nucl. Phys. A 697, 879 (2002); Nucl. Phys. A 707, 175 (2002)

20. V.P. Gonçalves, C.A. Bertulani, Phys. Rev. C 65, 054905 (2002)

21. S.R. Klein, J. Nystrand, R. Vogt, Phys. Rev. C 66, 044906 (2002)

22. S. Catani, M. Ciafaloni, F. Hautmann, Nucl. Phys. B 366, 135 (1991)

23. J. Collins, R. Ellis, Nucl. Phys. B 360, 3 (1991)

24. L. Gribov, E. Levin, M. Ryskin, Phys. Rep. 100, 1 (1983); E.M. Levin, M.G. Ryskin, Y.M. Shabelski, A.G. Shuvaev, Sov. J. Nucl. Phys. 53, 657 (1991)

25. G. Baur, K. Hencken, D. Trautmann, S. Sadovsky, Y. Kharlov, Phys. Rept. 364, 359 (2002)

26. V.P. Gonçalves, M.V. Machado, Eur. Phys. J. C 28, 71 (2003)

27. V.P. Gonçalves, M.V. Machado, Eur. Phys. J. C 29, 37 (2003)

28. V.P. Gonçalves, M.V. Machado, Eur. Phys. J. C 29, 271 (2003)

29. R. Nisius, Phys. Rep. 332, 165 (2000)

30. M. Glück, E. Reya, Phys. Lett. 79, 453 (1978)

31. K.J. Eskola, V.J. Kolhinen, C.A. Salgado, Eur. Phys. J. C 9, 61 (1999)
32. M. Gluck, E. Reya, A. Vogt, Eur. Phys. J. C 5, 461 (1998)

33. SMALL $x$ Collaboration, B. Anderson et al., Eur. Phys. J. C 25, 77 (2002)

34. A.V. Lipatov, V.A. Saleev, N.P. Zotov, Mod. Phys. Lett. A 15, 1727 (2000); Y.M. Shabelski, A.G. Shuvaev, hepph/0107106; M.G. Ryskin, A.G. Shuvaev, Y.M. Shabelski, Phys. Atom. Nucl. 64, 1995 (2001); S.P. Baranov et al., Eur. Phys. J. C 24, 425 (2002); H. Jung, Phys. Rev. D 65, 034015 (2002)

35. C. Brenner Mariotto, M.B. Gay Ducati, M.V.T. Machado, Phys. Rev. D 66, 114013 (2002)

36. L. Motyka, N. Timneanu, Eur. Phys. J. C 27, 73 (2003)

37. S.J. Brodsky, G.P. Lepage, P.B. Mackenzie, Phys. Rev. D 28, 228 (1983)

38. M. Glück, E. Reya, A. Vogt, Z. Phys. C 67, 433 (1995)

39. N. Armesto, Eur. Phys. J. C 26, 35 (2002)

40. V. Barone, E. Predazzi, High-energy particle diffraction (Springer-Verlag, 2002)

41. C.W. De Jager, H. De Vries, C. De Vries, Atom. Data Nucl. Data Tabl. 14, 479 (1974)

42. K. Golec-Biernat, M. Wüsthoff, Phys. Rev. D 60, 114023 (1999); Phys. Rev. D 59, 014017 (1998)

43. N. Armesto, M.A. Braun, Eur. Phys. J. C 20, 517 (2001)

44. V.P. Gonçalves, M.V.T. Machado, hep-ph/0307090

45. J. Bartels, S. Gieseke, C.F. Qiao, Phys. Rev. D 63, 056014 (2001) [Erratum D 65, 079902 (2002)]; J. Bartels, S. Gieseke, A. Kyrieleis, Phys. Rev. D 65, 014006 (2002); J. Bartels, D. Colferai, S. Gieseke, A. Kyrieleis, Phys. Rev. D 66, 094017 (2002)

46. S. Catani, M. Ciafaloni, F. Hautmann, Phys. Lett. B 242 , 97 (1990); Nucl. Phys. B 366, 135 (1991)

47. F. Gelis, J. Jalilian-Marian, Phys. Rev. D 66, 014021 (2002); Phys. Rev. D 66, 094014 (2002); Phys. Rev. D 67, 074019 (2003)

48. A. Krasnitz, R. Venugopalan, Nucl. Phys. B 557, 237 (1999); Phys. Rev. Lett. 84, 4309 (2000); 86, 1717 (2001); A. Krasnitz, Y. Nara, R. Venugopalan, Phys. Rev. Lett. 87, 192302 (2001); Nucl. Phys. A 717, 268 (2003); hep$\mathrm{ph} / 0305112$

49. H. Abramowicz, A.C. Caldwell, Rev. Mod. Phys. 71, 1275 (1999)

50. A.H. Mueller, Nucl. Phys. B 558, 285 (1999)

51. L. McLerran, R. Venugopalan, Phys. Rev. D 59, 094002 (1999); R. Venugopalan, Acta Phys. Polon. B 30, 3731 (1999)

52. A. Dumitru, L. McLerran, Nucl. Phys. A 700, 492 (2002)

53. A. Dumitru, J. Jalilian-Marian, Phys. Lett. B 547, 15 (2002); Phys. Rev. Lett. 89, 022301 (2002)

54. E. Ferreiro, E. Iancu, K. Itakura, L. McLerran, Nucl. Phys. A 710, 373 (2002)

55. E. Iancu, K. Itakura, L. McLerran, Nucl. Phys. A 724, $181(2003)$

56. A.H. Mueller, Nucl. Phys. A 724, 223 (2003)

57. Y.V. Kovchegov, Phys. Rev. D 61, 074018 (2000); E. Levin, K. Tuchin, Nucl. Phys. B 573, 833 (2000); A 691, 779 (2001); 693, 787 (2001); M. Braun, Eur. Phys. J. C 16, 337 (2000); K. Golec-Biernat, L. Motyka, A.M. Stasto, Phys. Rev. D 65, 074037 (2002)

58. A.H. Mueller, D.N. Triantafyllopoulos, Nucl. Phys. B 640 , 331 (2002); D.N. Triantafyllopoulos, Nucl. Phys. B 648, $293(2003)$

59. J. Raufeisen, J.C. Peng, Phys. Rev. D 67, 054008 (2003)

60. A.D. Martin, R.G. Roberts, W.J. Stirling, R.S. Thorne, Eur. Phys. J. C 4, 463 (1998) 